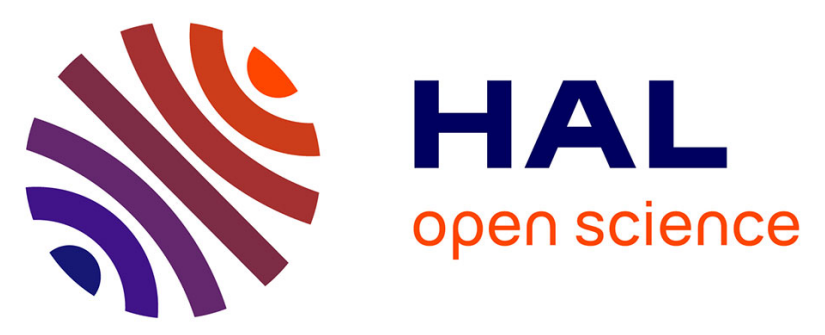

\title{
On the most convenient Mixed Strategies in a Mixed Strategist Dynamics Approach for Load Management of Electric Vehicle Fleets
}

Andres Ovalle, Seddik Bacha, Ahmad Hably, Kaustav Basu

\section{- To cite this version:}

Andres Ovalle, Seddik Bacha, Ahmad Hably, Kaustav Basu. On the most convenient Mixed Strategies in a Mixed Strategist Dynamics Approach for Load Management of Electric Vehicle Fleets. IECON 2016 - 42nd Annual Conference of the IEEE Industrial Electronics Society, Oct 2016, Florence, Italy. hal-01385564

\author{
HAL Id: hal-01385564 \\ https://hal.science/hal-01385564
}

Submitted on 21 Oct 2016

HAL is a multi-disciplinary open access archive for the deposit and dissemination of scientific research documents, whether they are published or not. The documents may come from teaching and research institutions in France or abroad, or from public or private research centers.
L'archive ouverte pluridisciplinaire HAL, est destinée au dépôt et à la diffusion de documents scientifiques de niveau recherche, publiés ou non, émanant des établissements d'enseignement et de recherche français ou étrangers, des laboratoires publics ou privés. 


\title{
On the most convenient Mixed Strategies in a Mixed Strategist Dynamics Approach for Load Management of Electric Vehicle Fleets
}

\author{
Andres Ovalle*^, Seddik Bacha*, Ahmad Hably*, Kaustav Basu* \\ ${ }^{*}$ Grenoble Electrical Engineering Laboratory (G2ELab), ${ }^{\star}$ Grenoble Image Parole Signal Automatique (Gipsa-Lab) \\ Université de Grenoble Alpes, France (andres.ovalle-villamil@g2elab.grenoble-inp.fr)
}

\begin{abstract}
This manuscript explores the selection of appropriate mixed strategies (MSs) in a Mixed Strategist Dynamics (MSD) application for load management of Plug-in Electric Vehicle (PEV) fleets. This selection is based on the convenience of PEV owners, aiming to choose those MSs that privilege early high (or fast) charging rates when it is possible. The previously published MSD and Maximum Entropy principle (MSD-MEP) approach is revised and illustrated with several examples, specially in the context of selection of MSs. This revision allows a wider understanding of the method, and aims to inspire new contributions on domains where distributed optimization methods are pertinent. Results obtained without any management structure are compared to those obtained with the MSD-MEP approach under different scenarios, where full sets of MSs and reduced sets of convenient MSs are applied. The performance of the method, using conveniently reduced sets of MSs, is tested with real historical active power measurements, provided by the SOREA utility grid company in the region of Savoie, France.

Index Terms-Plug-In Hybrid Electric Vehicles, Smart charging, Evolutionary Game theory, Distributed Optimization, Mixed Strategist Dynamics.
\end{abstract}

\section{INTRODUCTION}

Maturing and emerging technologies have boosted the markets of plug-in electric vehicles (PEVs) and plug-in hybrid electric vehicles in the recent years, in terms of battery capacities and autonomy. These technological advancements have impacts not only in the development of sustainable mobility systems [1], but also on the electrical infrastructure that provides power to these systems [2]-[5]. Connected PEVs represent distributed energy storage devices to the electricity distribution system, that can be employed to improve power quality and efficiency or reduce maintenance costs [6]. Nevertheless, without any smart management strategy handling charging infrastructures, distribution systems are prone to have problems such as heavy load unbalances, high load peaks and fast transitions, or transformers lifespan reduction [7]-[10].

Several approaches have been proposed in order to properly handle PEV fleets load. In particular, decentralized approaches are preferable because of the benefits the provide for owners autonomy [11]. In [12], a communication channel inspired approach is proposed, where each PEV load is divided in several packets. Each PEV asks for permissions in order to consume its programmed packets of load, according to the congestion of the system. Authors of [13] propose an approach for low penetration of PEVs, where each one maximizes its charging rates taking into account voltage and current constraints based on offline parameter computations. Uncertain final states of charge, sudden variations in power consumption heavily affecting batteries and transformers, and inflexible charging rates, are some of the drawbacks of these approaches.

Other authors formulate the load scheduling problem as $\mathrm{N}$ person potential games (a concept from game theory), where each PEV is a player [5], [14]. These techniques optimize a certain criteria by defining proper load schedules in a decentralized way. Some weak points in these approaches arise when the number of PEVs is not large enough, or when parameters (total PEV load, charger power limits, connection times) are not homogeneous. Moreover, these methods unfairly allocate the available resources. In [6], an evolutionary game dynamics approach is proposed based on the application of the Mixed strategist Dynamics (MSD) and the Maximum Entropy principle (MEP) in a multi-population model. This method handles the distributed PEVs load allocation problem in a fair way, reducing the impact on the grid, and taking into account social constraints from owners side. It takes advantage of the concept of Mixed Strategies (Ms) and applies it in order to define local sets of feasible solutions as convex hulls, where the local dynamics evolve.

In this paper, the previously published MSD-MEP approach of [6], is explored in terms of the selection of appropriate MSs. This selection is based on the convenience of PEV owners, aiming to choose those MSs that privilege early high (or fast) charging rates when it is viable. Several details and examples of the MSD-MEP method and the proposed appropriate selection of MSs are provided throughout the manuscript.

The paper is organized as follows. Section II provides a brief introduction to the MSD, with some examples clarifying the definition and usage of MSs. Section III summarizes the MSD-MEP application, and introduces illustrative examples. Section IV describes the role of MSs in the MSD approach. Besides, it describes the selection of MSs privileging early high charging rates, among a full set of MSs. Section V provides results under several illustrative and realistic scenarios. 

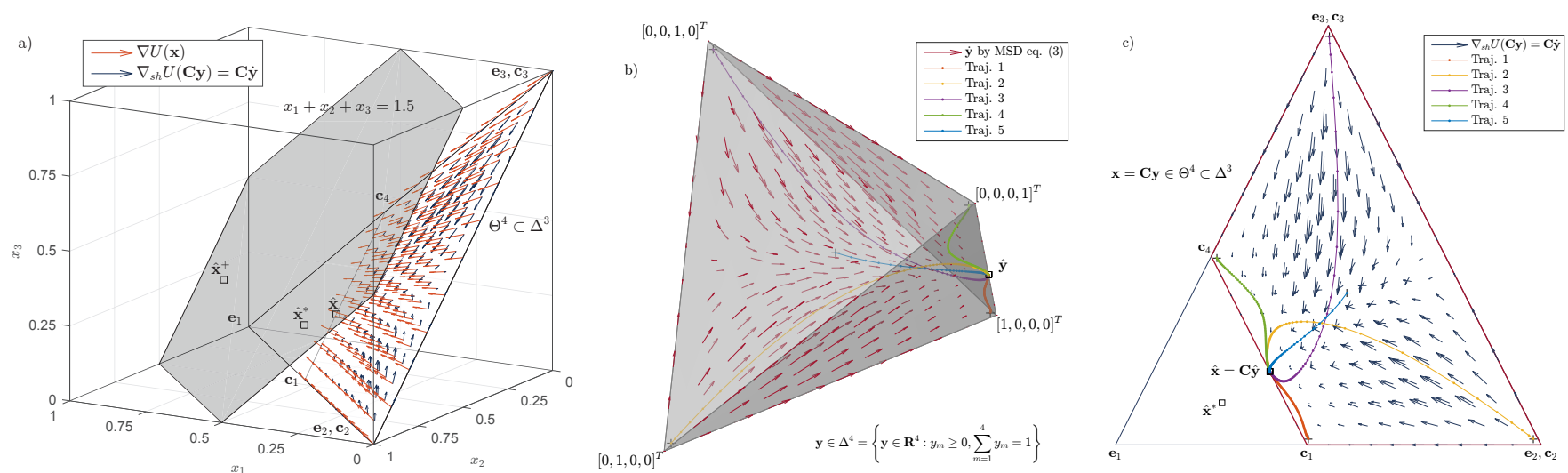

Fig. 1. a) Euclidean and Shahshahani gradient vector fields (scaled) for an example of potential function with domain $\mathbf{x} \in \mathbb{R}^{3}$. The feasible maximum $\hat{\mathbf{x}}$ lies in the subset $\Theta^{4}$ of the simplex $\Delta^{3}$, while unfeasible maxima $\hat{\mathbf{x}}^{*}$, and $\hat{\mathbf{x}}^{+}$lie in planes $\left\{x_{1}+x_{2}+x_{3}=1\right\}$, and $\left\{x_{1}+x_{2}+x_{3}=1.5\right\}$ respectively, in $\mathbb{R}^{3}$. b) Representation of the simplex $\Delta^{4} \in \mathbb{R}^{4}$ as a tetrahedron in $\mathbb{R}^{3}$, Shahshahani gradient vector field (scaled) in $\Delta^{4}$, and 5 different trajectories converging to the maximum. c) Equivalent Shahshahani gradient vector field, and equivalent 5 trajectories, as seen in $\Delta^{3}$. ( + : initial distributions, $\square$ : maxima)

Finally, Sect. VI gives a summary of conclusions, and section VII acknowledges some contributions to this work.

\section{Summary AND SOME DETAILS ON THE MSD}

As in [6], [15], [16], the MSD describes the evolution of the distribution of a population whose individuals are able to choose among an amount $M$ of MSs in a predefined set, depending on the payoff they get from each of them. These MSs are convex combinations of an original set of $K$ pure strategies, and the payoffs associated to MSs depend on those associated to pure strategies. The set of MSs can be represented by the vertices of a standard simplex in $\mathbb{R}^{M}$,

$$
\Delta^{M}=\left\{\mathbf{y} \in \mathbb{R}^{M}: y_{m} \geq 0, \sum_{m=1}^{M} y_{m}=1\right\},
$$

where every point $\mathbf{y}$ is a distribution. On the other hand, given a distribution $\mathbf{y} \in \mathbb{R}^{M}$ there is an associated distribution $\mathbf{x} \in$ $\mathbb{R}^{K}$ which can be found as,

$$
\mathbf{x}=\mathbf{C y},
$$

where the $M$ columns of $\mathbf{C}$ represent all the MSs of the predefined set. Given that each MS is a convex combination of the original set of $K$ pure strategies, each column $m=\{1, \cdots, M\}$ of $\mathbf{C}$ has $K$ elements $c_{k, m}$ such that, $c_{k, m} \geq 0, \forall k=\{1, \cdots, K\}$ and $\sum_{k=1}^{K} c_{k, m}=1$. Furthermore, a distribution given the original pure strategies can also be represented by a point $\mathbf{x}$ inside the simplex in $\mathbb{R}^{K}$,

$$
\Delta^{K}=\left\{\mathrm{x} \in \mathbb{R}^{K}: x_{k} \geq 0, \sum_{k=1}^{K} x_{k}=1\right\},
$$

where again each vertex represents a strategy, in this case, from the set of pure $K$ strategies. As it can be noticed, MSs are points inside the simplex $\Delta^{K}$, and distributions by MSs $\mathbf{y} \in \Delta^{M}$ have representations in terms of pure strategies as points $\mathbf{x} \in \Delta^{K}$ given by eq. (2). As it is described in [6], this is useful to define a subset $\Theta^{M} \subset \Delta^{K}$ of viable distributions $\mathbf{x}$ in terms of pure strategies, defined by,

$$
\Theta^{M}=\left\{\mathbf{x}=\mathbf{C y} \in \mathbb{R}^{K}: y_{m} \geq 0, \sum_{m=1}^{M} y_{m}=1\right\},
$$

which will be exemplified later in this section. The MSD is described in continuous time by,

$$
\dot{y}_{m}=y_{m}\left(\left(\mathbf{C}^{\mathrm{T}} \mathbf{f}(\mathbf{C y})\right)_{m}-\bar{g}(\mathbf{y})\right),
$$

where $\mathbf{f}(\mathbf{C y}):=\mathbf{f}(\mathbf{x})$ is a $K$ dimensional vector whose elements are the payoffs associated to pure strategies, given the population distribution $\mathbf{y}$ by MSs, and eq. (2). Correspondingly, vector $\mathbf{g}(\mathbf{y}):=\mathbf{C}^{\mathrm{T}} \mathbf{f}(\mathbf{C y})$ is an $M$ dimensional vector whose elements are the payoffs associated to MSs. Given the nature of the columns of $\mathbf{C}$, element $m$ of $\mathbf{g}$ can be interpreted as the expected value of using the probability mix of pure strategies given by the elements of column $m$. Term $\bar{g}(\mathbf{y})$ represents the weighted average value of payoffs.

When payoff functions for pure strategies are defined as the elements of the Euclidean gradient vector of a given potential function, it can demonstrated that eq. (5) represents a different type of gradient vector called Shahshahani gradient. Thus, un der the MSD, $\mathbf{y}$ evolves and converges to a local maximum $\hat{\mathbf{y}}$ for initial distributions in its neighborhood [6], [15]-[17].

In order to illustrate these properties, let us consider a potential function $U(\mathbf{x})=-\left(x_{1}-26 / 30\right)^{2}-\left(x_{2}-11 / 30\right)^{2}-$ $\left(x_{3}-8 / 30\right)^{2}$, for $\mathbf{x} \in \mathbb{R}^{3}$. Now, let us define the payoffs vector for pure strategies as the euclidean gradient vector of this potential function. The function $U(\mathbf{x})$ has a global maximum in $\hat{\mathbf{x}}^{+}=[26 / 30,11 / 30,8 / 30]$, which is a point in the plane $\left\{x_{1}+x_{2}+x_{3}=1.5\right\} \in \mathbb{R}^{3}$. However, if the feasible set is constrained to points $\mathbf{x} \in \Delta^{3}=\left\{x_{1} \geq 0, x_{2} \geq\right.$ $\left.0, x_{3} \geq 0, x_{1}+x_{2}+x_{3}=1\right\}$, then the feasible maximum is $\hat{\mathbf{x}}^{*}=[7 / 10,2 / 10,1 / 10]$. If the feasible set is even more constrained to $\mathrm{x} \in \Theta^{4}=\left\{0 \leq x_{1} \leq 0.55, x_{2} \geq 0, x_{3} \geq\right.$ $\left.0, x_{1}+x_{2}+x_{3}=1.5\right\}$, then the feasible maximum is now 

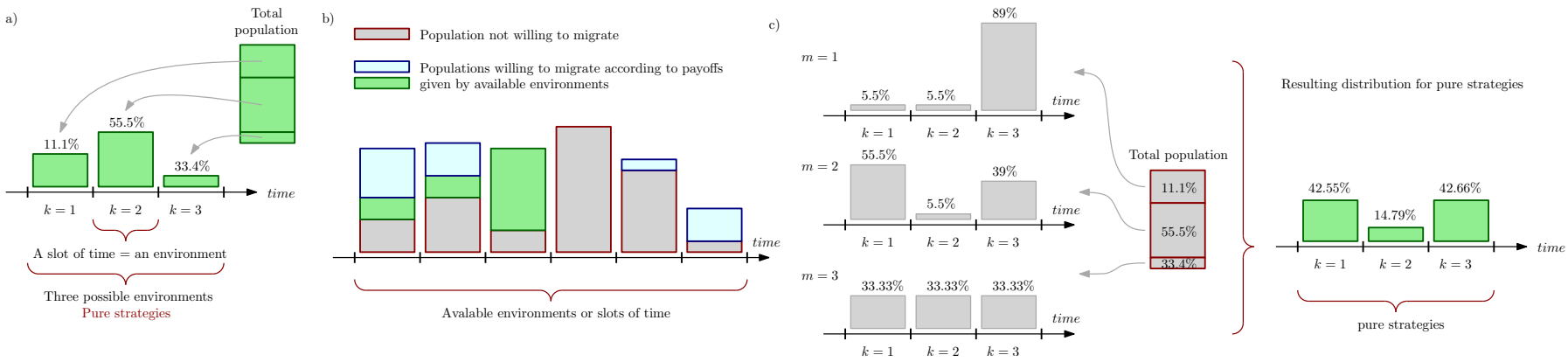

Fig. 2. a) Example of a distribution of a single population (PEV load) in three environments (pure strategies, or slots of time). b) Example of a multipopulation model, with populations willing to migrate (PEV load) through available environments, and sedentary populations (Base forecast load). c) Example of distribution of a single population (PEV load) among three predefined MSs, and the resulting distribution among pure strategies.

$\hat{\mathbf{x}}=[22 / 40,11 / 40,7 / 40]$. It should be noticed that $\Theta^{4}$ can also be defined by the convex hull of eq. (4), and the MSs of,

$$
\mathbf{C}=\left[\begin{array}{cccc}
0.55 & 0 & 0 & 0.55 \\
0.45 & 1 & 0 & 0 \\
0 & 0 & 1 & 0.45
\end{array}\right]
$$

Both the unfeasible maxima and the feasible maximum are shown in Fig. 1(a). This example illustrates a comparison between the Euclidean gradient and the Shahshahani gradient vectors. For several points $\mathbf{x}$ in the feasible set $\Theta^{4}$, Fig. 1(a) shows the vector fields of both gradients. As it can be observed, the euclidean gradient vector $\nabla U(\mathbf{x})$ points to the plane $\left\{x_{1}+x_{2}+x_{3}=1.5\right\}$ which contains the unconstrained maximum $\hat{\mathbf{x}}^{+}$. This happens because the euclidean gradient points in the direction of maximal increase of the potential function $U(\mathbf{x})$. On the other hand, the Shahshahani gradient vector $\nabla_{s h} U(\mathbf{C y})=\mathbf{C} \dot{\mathbf{y}}$ lies on the tangent space $T_{\mathbf{x}} \Delta^{3}=$ $\left\{x_{1}+x_{2}+x_{3}=0\right\}$ of the simplex $\Delta^{3}$, which in words means that its tail (i.e. $\mathbf{x}$ ) and tip (i.e. $\mathbf{x}+\mathbf{C} \dot{\mathbf{y}}$ ) both lie on the plane $\left\{x_{1}+x_{2}+x_{3}=1\right\} \supset \Delta^{3}$ [15], [17], [18].

The sample points $\mathbf{x} \in \Delta^{3}$ on Fig. 1(a) are obtained from uniformly distributed sample points $\mathbf{y} \in \Delta^{4}$, by applying eq. (2). On Fig. 1(b), it is possible to check the original sample points $\mathrm{y} \in \Delta^{4}$ and the corresponding vector field of the Shahshahani gradient $\dot{\mathbf{y}}$ for the potential function of the example. Fig. 1(c) shows a profile view of the simplex $\Delta^{3}$ and the subset $\Theta^{4}$. It also illustrates the vector field of $\dot{\mathbf{y}}$ within the convex hull given by the MSs of $\mathbf{C}$, i.e. $\dot{\mathbf{x}}=\mathbf{C} \dot{\mathbf{y}}$.

In Fig. 1(b) it is possible to check 5 different trajectories, for the evolution of the population, starting from different initial distributions $\mathbf{y}$ within $\Delta^{4}$. The equivalent trajectories in $\Delta^{3}$ obtained with eq. (2), are plotted in Fig. 1(c) as well. It is possible to check that trajectories converge to the feasible optimal.

These characteristics are exploited in [6] in the design of a methodology for the distributed load management of electrical vehicle fleets. In the next section, this methodology and the proposed analogies are summarized.

\section{SUMMARY OF THE MSD APPROACH FOR A PEV FLEET LOAD MANAGEMENT}

The method proposed [6] employs multiple analogies that will be summarized an illustrated in this section. In a first place, pure strategies are considered to be all the possible slots of time (hour steps) where PEVs can distribute their energy demand in order to fully charge their batteries. An example is shown on on Fig. 2(a), where a given population is distributed among three environments: $11.1 \%$ on the first, $55.5 \%$ on the second, and $33.4 \%$ on the third. Moreover, slots of time have previously allocated load corresponding to the forecasted demand of the transformer. In this scenario, PEVs load and forecasted load are both interpreted as populations, willing and not willing to migrate (nomad and sedentary populations) among environments (slots of time or pure strategies). An example illustrates these analogies on Fig. 2(b), where a sedentary population and two nomad populations are distributed in six environments (in the fourth environment there is only a portion of the sedentary population). The sedentary population represents the forecasted load profile for $K=6$ time slots (hours for instance), while the two nomad populations represent the load of two PEVs. These two nomad population distributions over the six environments, will evolve in order to maximize their weighted average payoffs.

The MSD-MEP approach proposed in [6] is a multipopulation model, where each single nomad population distribution evolves taking into account the portability of the environments and the payoffs they provide. These payoffs are defined by the trade-off between two measurements, a local load distribution entropy measurement and a total load distribution entropy measurement, given by,

$$
f_{k}^{i}\left(x_{k}^{i}\right)=-\alpha \ln \left(\frac{l_{k}}{\mu}+\sum_{j=1}^{J} \frac{x_{k}^{j}}{\mu}\right)-(1-\alpha) \ln \left(\frac{x_{k}^{i}}{\Gamma^{i}}\right)-1
$$

where, $\alpha^{i}$ is the trade-off factor $\left(0 \leq \alpha^{i} \leq 1\right)$ defined by the owner of PEV $i, \alpha$ is the mean of all the trade-off factors, $l_{k}$ is the forecasted load for time slot $k, x_{k}^{i}$ is the load from PEV $i$ allocated to time slot $k$. On the other hand, the total load $\mu$ 


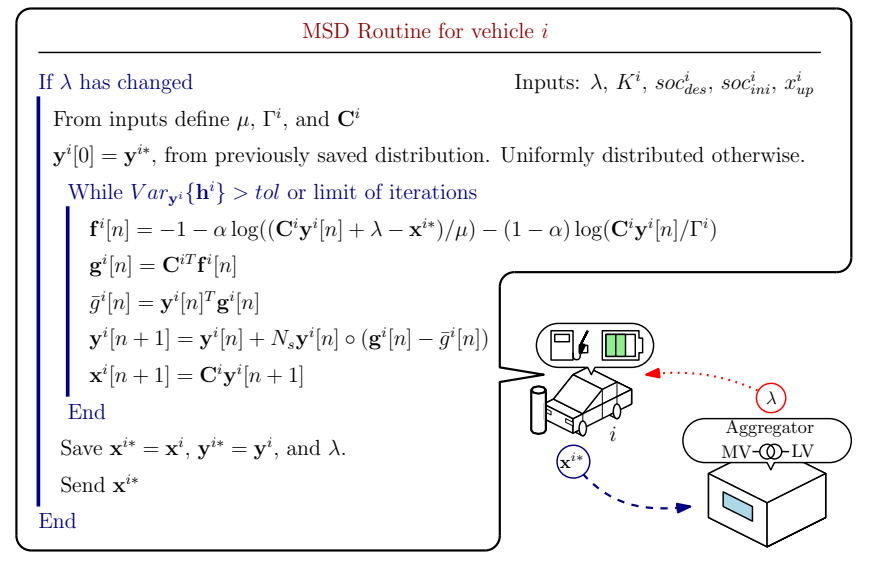

Fig. 3. Description of a local MSD routine [6]. Operator $\mathbf{a} \circ \mathbf{b}$ represents the entry-wise product among vectors $\mathbf{a}$ and $\mathbf{b}$. In this manuscript, the trade-off factor $\alpha$ is common to all single populations.

including both forecast and PEVs, and the total local load $\Gamma^{i}$ for PEV $i$, are given by,

$$
\mu=\sum_{k=1}^{K} \lambda_{k}, \quad \Gamma^{i}=\frac{s o c_{d e s}^{i}-s o c_{i n i}^{i}}{\tau},
$$

where $\lambda_{k}=\left(l_{k}+\sum_{j=1}^{J} x_{k}^{j}\right)$ is the total load at time slot $k$, $s o c_{i n i}^{i}$ and $s o c_{d e s}^{i}$ are the initial and desired states of charge for PEV $i$, and $\tau$ is the length of time slots (hours). It should be noticed that total and individual PEV load distributions lie in simplices like the one of eq. (3), given by,

$$
\begin{gathered}
\Delta_{\mu}^{K}=\left\{\lambda \in \mathbb{R}^{K}: \lambda_{k} \geq 0, \sum_{k=1}^{K} \lambda_{k}=\mu\right\}, \\
\Delta_{\Gamma^{i}}^{K^{i}}=\left\{\mathbf{x}^{i} \in \mathbb{R}^{K^{i}}: x_{k}^{i} \geq 0, \sum_{k=1}^{K^{i}} x_{k}^{i}=\Gamma^{i}\right\} .
\end{gathered}
$$

Each individual population distribution evolves such that the trade-off is maximized. The trade-off parameter is defined by owners In the extreme case where $\alpha=1$, each local evolution is such that the total load reaches a distributtion as uniform as possible among the environments. In the other extreme case, where $\alpha=0$, each local evolution is such that the local load of the PEV is distributed as uniformly as possible among the environments without taking into account the sedentary population, or other PEVs populations. The evolution of each population distribution is locally controlled by each PEV which has an associated MSD routine like the one presented in Fig. 3. Further details on the MSD routine are provided in [6].

\section{THE MOST CONVENIENT MSS FOR THE APPROACH}

In order to illustrate the role of MSs in this approach, let us consider the example of Fig. 2(c). In this example the population of a PEV is going to be split among three predefined MSs. These MSs are possible ways of distributing the PEV load in the available environments, the first MS

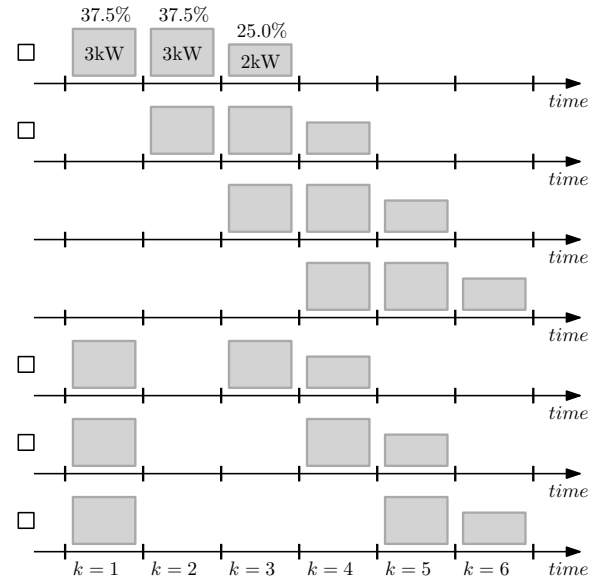

Fig. 4. Reduced set of the convenient MSs, for a PEV with an energy demand of $8 \mathrm{kWh}, K^{i}=6$ hours, and a $3 \mathrm{~kW}$ charger. The full set has $M^{i}=60$ MSs. ( $\square$ : Most convenient MSs privileging early high charging rates.)

$(m=1)$ pre-defines $5.5 \%$ of the population allocated in the first environment, $5.5 \%$ in the second one, and $89 \%$ on the third; the second MS $(m=2)$ pre-defines $55.5 \%, 5.5 \%$, and $39 \%$ for each environment; and the last MS $(m=3)$ pre-defines uniformly $33.3 \%$ for each environment. These MSs define a matrix $\mathbf{C}$ as in eq. (2). In this example, the PEV population is distributed among these three MSs by $11.1 \%$ for $m=1,55.5 \%$ for $m=2$, and $33.4 \%$ for $m=3$. This distribution per MSs can be summarized in $\mathbf{y}=[0.111,0.555,0.334]^{\mathrm{T}}$, and applying eq. (2), the total load is divided by $42.55 \%$ for $k=1,14.79 \%$ for $k=2$, and $42.66 \%$ for $k=3$, or $\mathbf{x}=[0.4255,0.1479,0.4266]^{\mathrm{T}}$.

In this approach, MSs are defined based on the limitation of the population that can be assigned to an environment, i.e. the maximum instantaneous power allowed by the chargers. Based on the constraints $0 \leq x_{k}^{i} \leq x_{u p}^{i}$, the total number of MSs was defined by,

$$
M^{i}=\frac{K^{i} !}{\left(\gamma^{i}-1\right) !\left(K^{i}-\gamma^{i}\right) !},
$$

where $K^{i}$ is the total number of pure strategies for PEV $i$, and $\gamma^{i}$ is the minimum amount of slots of time needed to allocate the PEV load taking into account the charger limitation of power. As it was shown in [6], this number can be very large depending on the problem. However, in this manuscript the idea of only using the most interesting MSs is further explored.

In order to illustrate the concept of the most convenient MSs in this approach, let us consider a PEV with an energy demand of $8 \mathrm{kWh}$. This PEV has to charge its battery in $K^{i}=6$ hours with a $3 \mathrm{~kW}$ charger. The minimum amount of environments needed is then $\gamma^{i}=3$, and the total amount of MSs that represent completely the feasible set is $M^{i}=60$. Nevertheless, some of the most interesting MSs can be selected as those which are more convenient for the PEV owners. For instance, in Fig. 4, for the same example, 7 out of the 60 MSs are plotted. These MSs are those that charge the battery as fast 

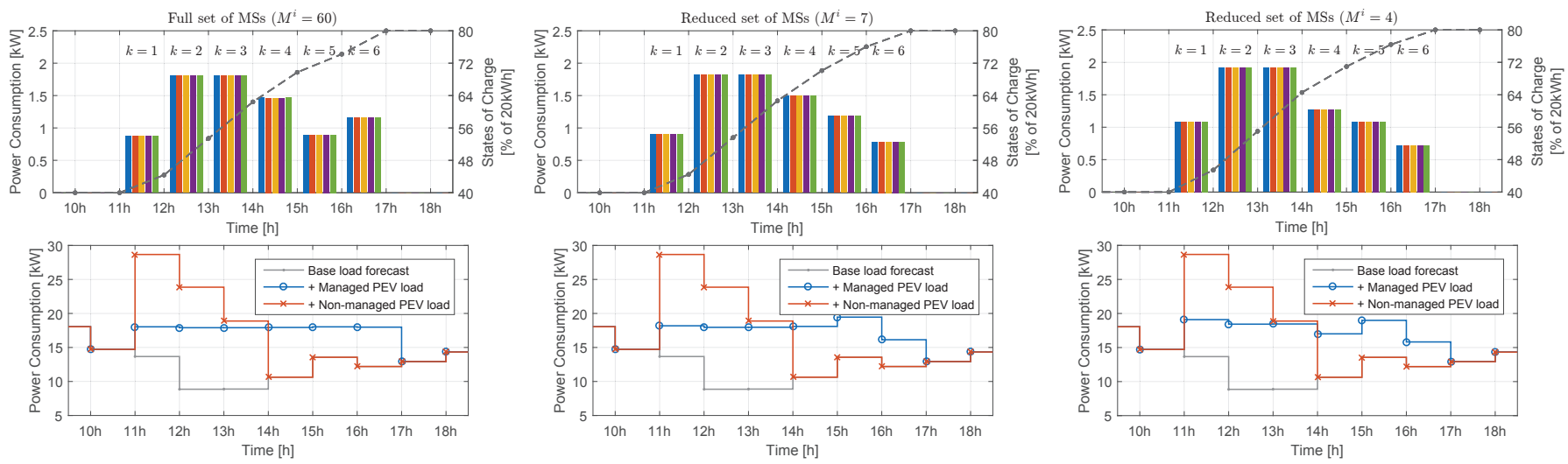

Fig. 5. Example of 5 PEVs, all of them with energy demands of $8 \mathrm{kWh}, K^{i}=6$ hours to charge their batteries, and $3 \mathrm{~kW}$ chargers. Left: All of them using full sets of 60 MSs. Middle: All of them using reduced sets of only 7 convenient MSs. Right: All of them using reduced sets of 4 of the most convenient MSs, privileging early high charging rates. Upper: final PEV load distributions and state of charge profiles of the 5 PEVs, for the three cases. Lower: final total load distributions, for the three cases, compared to the non-managed case.
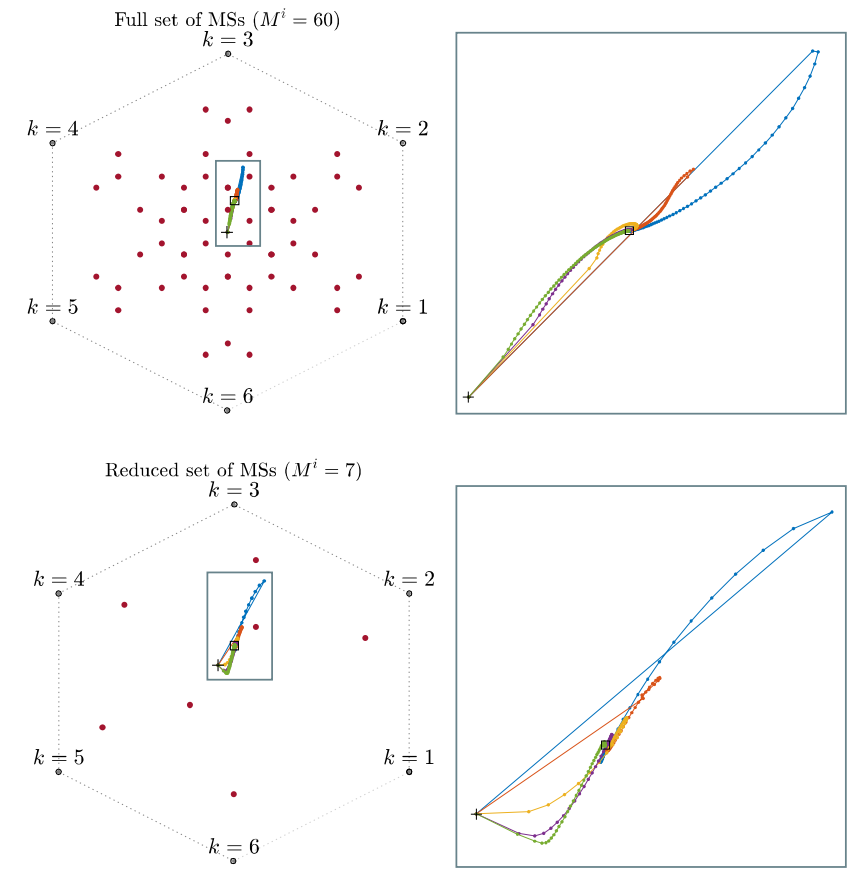

Fig. 6. Representation of the evolution of the 5 distributions as a convex combination of the vertices of an hexagon (6 pure strategies), and zoom on the trajectories (right). Red dots represent the MSs used in the respective case. Top: Full set of MSs. Bottom: Set of 7 convenient MSs. (+: initial distributions, $\square$ : final distributions)

as the charger allows it, and those that apply full charging power during the first hour. The idea behind selecting these MSs consists in diving the total load of each PEV among only the convenient MSs and neglect other MSs that do not benefit the PEV owners. At the end, as it was explained with the example on Fig. 2(c), the final distributions are linear convex combinations of the chosen MSs. Consequently selecting only those MSs having a positive effect for owners, will result in final load distributions more beneficial for them, while grid services are still provided by PEVs as much as possible.

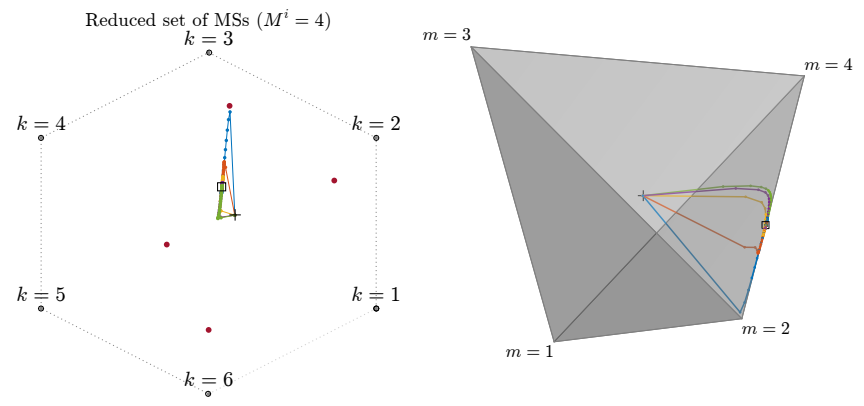

Fig. 7. Similar representation to that of Fig. 6, for the evolution of load distributions of the $5 \mathrm{PEVs}$ using the 4 most convenient MSs privileging early high charging rates. ( + : initial distributions, $\square$ : final distributions)

\section{RESUlts}

Firstly, 5 PEVs are considered to be charged in a residential grid between $11 \mathrm{~h}$ and $17 \mathrm{~h}$. These PEVs are connected to chargers limited in nominal power to $3 \mathrm{~kW}$. In these conditions, they have 6 hours to consume $8 \mathrm{kWh}$ in order to reach a desirable state of charge (soc) (initially they have $40 \%$ and they have to reach $80 \%$ of their storage capacities of $20 \mathrm{kWh}$ ). Thus the total amount of MSs for each PEV is again $M^{i}=60$ like in the example of the previous section. In Fig. 5, it is possible to observe the final load distributions obtained by the MSD approach in three cases: (Case 1) all the 60 MSs are used, (Case 2) only the 7 convenient MSs of Fig. 4 are used, and (Case 3) only the 4 most convenient MSs are used (the first two and the last two MSs in Fig. 4). The mean trade-off factor $\alpha$ is assumed to reach $\alpha=0.99$, given certain effective incentive policies from grid managers for PEV owners.

As it can be observed on the upper diagrams on Fig. 5, on each one of the three cases, all the PEVs converge to common final local load distributions. As it can be expected, using reduced sets of MSs still maintains the resource allocation fairness property of the MSD approach. Observing the lower diagrams on Fig. 5, when full sets of MSs are employed, 

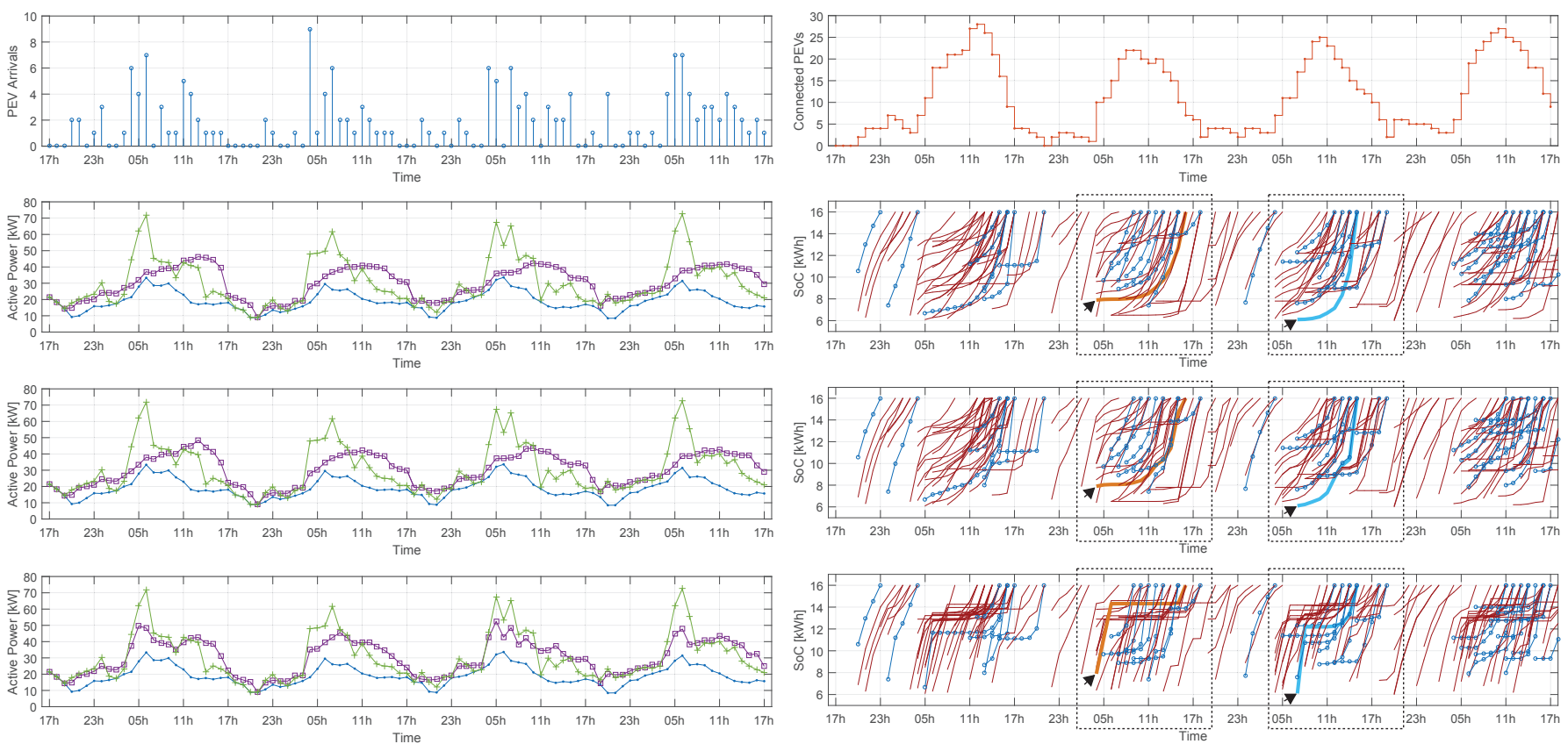

Fig. 8. From top to bottom: 1) Profiles of arrivals and connected PEVs throughout the evaluation time. 2) Load forecast (blue dots), PEV aggregated load without management (green +), PEV aggregated load with MSD and full sets of MSs (purple $\square$ ), and corresponding state of charge profiles (red for 3.3kW, and blue $\circ$ for $7.5 \mathrm{~kW}$ chargers). 3) Similar profiles, for MSD with reduced sets of convenient MSs. 4) Similar profiles, for MSD with reduced sets of the most convenient MSs, for early high charging rates. Bold line state of charge profiles: examples of 2 PEVs and the effect of convenient MSs.

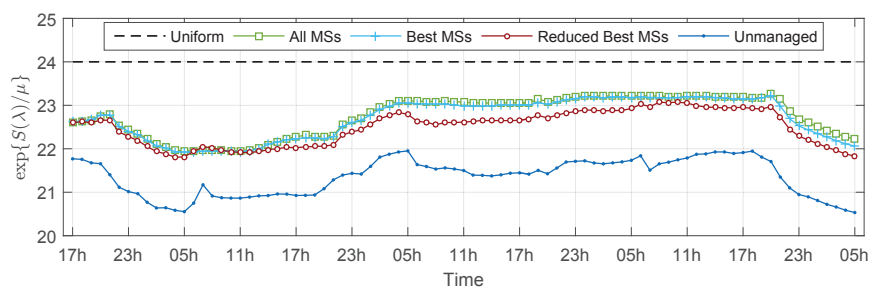

Fig. 9. Entropy measurements of total load distributions $\lambda$ (Forecast + PEVs), for the unmmanaged case, the ideal case (uniform distribution), and for the MSD approach with full sets of MSs, and convenient MSs. Measurements are taken each hour on three of the four study days (with 24 hours horizons).

the final total load distribution is almost flat, while for the cases with reduced sets of MSs, the final reached total load is more uneven. This happens because the local load distributions evolve such that they try to reach a maximum total load distribution entropy (a total load profile as flat as possible) with only the available MSs. On the other hand, it can be observed that reduced sets of MSs privilege early high charging rates, specially on the third case where the first three hours show increased charging rates compared to the other cases.

Figs. 6 and 7 illustrate the evolution of the convex combinations for the three cases. They show the mapping of the sets of MSs which are convex combinations as well. In these figures, each vertex represents a pure strategy or an environment (a slot of time $k$ ), and red points inside represent the corresponding MSs. It is also possible to check how the 5 load distributions evolve and converge to the same distribution. Given that the third case has a set of only four MSs, the 5 load distributions

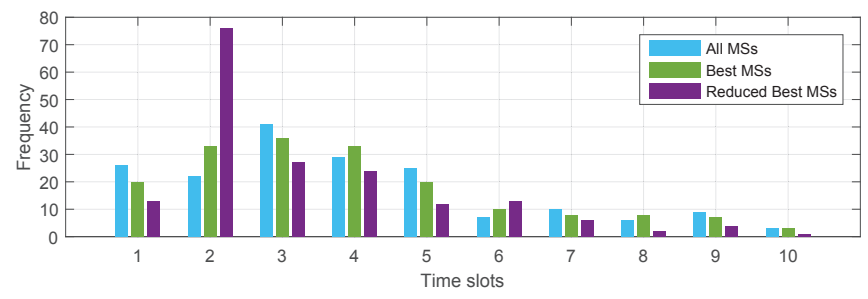

Fig. 10. Observed frequencies for the number of time slots required to overpass $50 \%$ of the energy needed. Frequencies corresponds to amounts of PEVs during the four study days. The MSD is tested using full sets of MSs, Best MSs, and Reduced Best MSs.

evolution can be more easily illustrated in the simplex $\Delta^{4}$ (a tetrahedron in $\mathbb{R}^{3}$ ) as it is show in Fig. 7.

On the other hand, a realistic scenario is presented, using real active power measurements of a distribution transformer, from the SOREA utility grid company, in the region of Savoie, France. These measurements correspond to mostly office buildings. In this case, the arrival and departure of several PEVs is studied in a random way, following a poisson model with variable rate of arrivals (changing with time) and variable connection times. The highest rate of arrivals is $5 \mathrm{PEVs} / \mathrm{h}$ around $05 \mathrm{~h}$ and it decays up to $0.5 \mathrm{PEVs} / \mathrm{h}$ at $04 \mathrm{~h}$ the next day. Vehicle battery capacities are defined to be $20 \mathrm{kWh}$. Chargers are randomly chosen to have limits of power of $3.3 \mathrm{~kW}$ with a probability of $80 \%$, and $7.5 \mathrm{~kW}$ with a probability of $20 \%$. For reducing impact on batteries lifespan, states of charge are constrained between $30 \%-80 \%$. The mean trade-off factor $\alpha$ is assumed to reach $\alpha=0.9$, given incentive 
policies from grid managers for PEV owners.

Fig. 8 shows the total load distribution using full sets of MSs, reduced sets of convenient MSs like those of Fig. 4, and reduced sets of only the most convenient MSs privileging early high charging rates. It is worth noting that the peak of connected vehicles is coincident with the peak of the base load demand, which makes a management indispensable. Despite the degradation observed in the final total load distributions with the reduction of the amount of used MSs, the proposed MSD approach with only convenient MSs, is still able to handle PEVs' load properly. Fairness, PEV owners' convenience, and impact reduction are still achieved. Taking into account that entropy of the total load distribution is given by $S(\lambda)=-\sum_{k=1}^{K} \lambda_{k} \ln \left(\lambda_{k} / \mu\right)$ [6], entropy measurements were taken for each hour step of the first three days of the scenario on Fig. 8. Using horizons of $K=24$ hours, the results are presented on Fig. 9 for the ideal case (uniform total load distribution), the unmanaged case, and the managed case using MSD-MEP with: full sets of MSs, Best MSs, and Reduced Best MSs. It is possible to observe that in terms of total load distribution entropy maximization, MSD-MEP with full sets of MSs achieves the closest entropy measurements to the ideal case (uniform distribution). It can be observed as well that the worst measurements correspond to the unmanaged case, as it is expected. The most important thing to highlight is the closeness of the entropy measurements for the MSDMEP using full and reduced sets of MSs, proving that the the proposed MSD approach with only convenient MSs, is still able to handle PEVs' load properly.

State of charge profiles on Fig. 8 show how owners benefit from the most convenient MSs. In particular, profiles in bold lines (marked with arrows) are useful to check how the use of the most convenient MSs affect charging rates, allowing high consumption as early as possible. Reduced sets of the most convenient MSs allow to take advantage from fast charging infrastructures while the impact on the transformers is still reduced, as it is observed with the entropy maximization measurements on Fig. 9. Additionally, Fig. 10 shows the frequency distributions of slots of time required to overpass $50 \%$ of the required energy, for all the PEVs during the four days. It can be observed that these frequency distributions are similar for full sets of MSs and sets of Best MSs, with mean values of 4.96 and 4.92 slots of time, respectively. However, with sets of Reduced Best MSs early fast charging rates are privileged, showing a reduction of the mean value to 3.28 slots of time, and a concentration of more than 70 vehicles (over a total of 178) using only 2 slots of time to overpass the $50 \%$.

\section{CONCLUSION}

This paper explores the selection of appropriate MSs in a MSD application for load management of PEV fleets. The MSD approach, using reduced sets of the most convenient MSs, privileging early high charging rates, is still able to handle the PEVs' load properly. Besides, fairness, PEV owners' convenience, and impact reduction are still achieved by the approach. As it has been studied, the most convenient
MSs allow to take advantage from fast charging infrastructures while the impact on the transformers is still reduced, compared to the case without management. Furthermore, this wider revision on the MSD approach, the definition and selection of MSs, and the illustration of Shahshahani gradient concepts, motivates the exploration and conception of new applications.

\section{ACKNOWLEDGMENT}

This work was supported by the ARC Energies Région Rhône-Alpes, and the Project ANR PARADISE, France.

\section{REFERENCES}

[1] M. Helmbrecht, C. Olaverri-Monreal, K. Bengler, R. Vilimek, and A. Keinath, "How electric vehicles affect driving behavioral patterns," IEEE Intelligent Transportation Systems Magazine, vol. 6, no. 3, pp. 22-32, Fall 2014.

[2] L. Jian, H. Xue, G. Xu, X. Zhu, D. Zhao, and Z. Shao, "Regulated charging of plug-in hybrid electric vehicles for minimizing load variance in household smart microgrid," IEEE Trans. Ind. Elec., vol. 60, no. 8, pp. 3218-3226, Aug 2013.

[3] A. Ovalle, A. Hably, and S. Bacha, "Optimal management and integration of electric vehicles to the grid: Dynamic programming and game theory approach," in ICIT 2015 - International Conference on Industrial Technology, March 2015.

[4] S. Sarabi, A. Bouallaga, A. Davigny, B. Robyns, V. Courtecuisse, Y. Riffonneau, and M. Regner, "The feasibility of the ancillary services for vehicle-to-grid technology," in European Energy Market (EEM), 2014 11th International Conference on the, May 2014.

[5] H. K. Nguyen and J. B. Song, "Optimal charging and discharging for multiple phevs with demand side management in vehicle-to-building," Journal Comm. and Networks, vol. 14, no. 6, pp. 662-671, Dec 2012.

[6] A. Ovalle, J. Fernandez, A. Hably, and S. Bacha, "An electric vehicle load management application of the mixed strategist dynamics and the maximum entropy principle," IEEE Transactions on Industrial Electronics, vol. 63, no. 5, pp. 3060-3071, May 2016.

[7] K. Dyke, N. Schofield, and M. Barnes, "The impact of transport electrification on electrical networks," IEEE Trans. Ind. Electron., vol. 57, no. 12 , pp. 3917-3926, Dec 2010.

[8] H. Turker, S. Bacha, D. Chatroux, and A. Hably, "Low-voltage transformer loss-of-life assessments for a high penetration of plug-in hybrid electric vehicles (PHEVs)," IEEE Trans. Power Del., vol. 27, no. 3, pp. 1323-1331, July 2012.

[9] H. Turker, S. Bacha, and A. Hably, "Rule-based charging of plugin electric vehicles (PEVs): Impacts on the aging rate of low-voltage transformers," IEEE Trans. Power Del., vol. 29, no. 3, pp. 1012-1019, June 2014.

[10] K. Clement-Nyns, E. Haesen, and J. Driesen, "The impact of charging plug-in hybrid electric vehicles on a residential distribution grid," IEEE Trans. Power Syst., vol. 25, no. 1, pp. 371-380, Feb 2010.

[11] Y. He, B. Venkatesh, and L. Guan, "Optimal scheduling for charging and discharging of electric vehicles," IEEE Trans. Smart Grid, vol. 3, no. 3, pp. 1095-1105, Sept 2012.

[12] P. Rezaei, J. Frolik, and P. Hines, "Packetized plug-in electric vehicle charge management," IEEE Trans. Smart Grid, vol. 5, no. 2, pp. 642 650, March 2014.

[13] P. Richardson, D. Flynn, and A. Keane, "Local versus centralized charging strategies for electric vehicles in low voltage distribution systems," IEEE Trans. Smart Grid, vol. 3, no. 2, pp. 1020-1028, June 2012.

[14] Z. Ma, D. Callaway, and I. Hiskens, "Decentralized charging control of large populations of plug-in electric vehicles," IEEE Trans. Control Syst. Technol., vol. 21, no. 1, pp. 67-78, Jan 2013.

[15] J. Hofbauer and K. Sigmund, The Theory of Evolution and Dynamical systems. Cambridge University Press, 1988.

[16] — Evolutionary Games and Population Dynamics. Cambridge University Press, 1998.

[17] M. Harper, "Information geometry and evolutionary game theory," CoRR, vol. abs/0911.1383, 2009.

[18] G. Lebanon, "Metric learning for text documents," IEEE Trans. Pattern Anal. Mach. Intell., vol. 28, no. 4, pp. 497-508, April 2006. 